\title{
How well is our universe described by an FLRW model?
}

\author{
Stephen R. Green ${ }^{1}$ and Robert M. Wald ${ }^{2}$ \\ 1 Department of Physics, University of Guelph \\ Guelph, Ontario N1G 2W1, Canada \\ ${ }^{2}$ Enrico Fermi Institute and Department of Physics, University of Chicago \\ 5640 South Ellis Avenue, Chicago, Illinois 60637, USA
}

E-mail: sgreen04@uoguelph.ca and rmwa@uchicago.edu

\begin{abstract}
Extremely well! In the $\Lambda \mathrm{CDM}$ model, the spacetime metric, $g_{a b}$, of our universe is approximated by an FLRW metric, $g_{a b}^{(0)}$, to about 1 part in $10^{4}$ or better on both large and small scales, except in the immediate vicinity of very strong field objects, such as black holes. However, derivatives of $g_{a b}$ are not close to derivatives of $g_{a b}^{(0)}$, so there can be significant differences in the behavior of geodesics and huge differences in curvature. Consequently, observable quantities in the actual universe may differ significantly from the corresponding observables in the FLRW model. Nevertheless, as we shall review here, we have proven general results showing that - within the framework of our approach to treating backreaction - the large matter inhomogeneities that occur on small scales cannot produce significant effects on large scales, so $g_{a b}^{(0)}$ satisfies Einstein's equation with the averaged stress-energy tensor of matter as its source. We discuss the flaws in some other approaches that have suggested that large backreaction effects may occur. As we also will review here, with a suitable "dictionary," Newtonian cosmologies provide excellent approximations to cosmological solutions to Einstein's equation (with dust and a cosmological constant) on all scales. Our results thereby provide strong justification for the mathematical consistency and validity of the $\Lambda \mathrm{CDM}$ model within the context of general relativistic cosmology.
\end{abstract}




\section{Introduction}

Modern cosmology is based on the assumption of a background FLRW model with small perturbations. With only six free parameters, the resulting $\Lambda$ CDM model successfully accounts for all cosmological data, including the CMB and its anisotropies, structure formation and galactic dynamics, redshift-luminosity relations, and the nucleosynthesis of light elements. It stands as a truly remarkable achievement of modern physics.

Nevertheless, there are grounds for questioning the validity - and, perhaps, even the mathematical consistency - of using the $\Lambda$ CDM model to describe our universe. The observed density contrasts in our universe are huge on scales of $\sim 1 \mathrm{Mpc}$ and smaller, and certainly cannot be described as "small perturbations" of an FLRW model in the usual sense. It might therefore appear that some sort of "averaging" over the small scale inhomogeneities must be used in order to even define the FLRW background model that is supposed to represent our universe in a $\Lambda$ CDM model. If one then succeeds in defining an averaged FLRW model, how does one know that its dynamics will be properly described by the dynamical equations applicable to an exact FLRW model? Therefore, it is appropriate to ask the question posed as the title of this paper: "How well is our universe described by an FLRW model?"

The issue of how best to approximate our lumpy universe by an FLRW model - the "fitting problem" - was discussed in classic papers of Ellis [1] and Ellis and Stoeger [2]. Ellis envisioned defining an operator that would take an inhomogeneous metric and stress-energy tensor into successively smoother and smoother versions (see figure 1). Given that the Einstein equation holds at the most inhomogeneous scale (his "Scale $1 ")$, Ellis raised the very important question of whether the Einstein equation holds at the level of the large scale averaged metric and stress-energy tensor. The averaged Einstein equation is

$$
G_{a b}\left(g^{(0)}\right)+\Lambda g_{a b}^{(0)}=8 \pi\left(T_{a b}^{(0)}+t_{a b}^{(0)}\right) .
$$

Here $g_{a b}^{(0)}$ and $T_{a b}^{(0)}$ represent, respectively, the large scale averaged metric and stressenergy tensor, and $t_{a b}^{(0)}$ is the average of the (nonlinear) contributions to Einstein's equation arising from the departures of the actual metric and stress-energy tensor from

$g_{a b}^{(0)}$ and $T_{a b}^{(0)}$. Thus, $t_{a b}^{(0)}$ represents the backreaction effects of the inhomogeneities on the large scale averaged metric, and we will refer to it as the effective stress-energy tensor produced by the inhomogeneities. The issues raised in the previous paragraph then reduce to the following: (i) How does one define the FLRW quantities $g_{a b}^{(0)}$ and $T_{a b}^{(0)}$ for the actual universe? (ii) Is $t_{a b}^{(0)}$ negligibly small?

One of the main points that we wish to emphasize in this article is that the first question has an extremely simple answer. As we shall elucidate further below, it is akin to asking how to represent the surface of a real ball bearing by a perfect sphere. The surface of a real ball bearing is extremely close to being a perfect sphere - despite significant imperfections that can be seen under a microscope - and it is therefore easily represented by a perfect sphere. Similarly, the actual metric, $g_{a b}$, of our universe is 


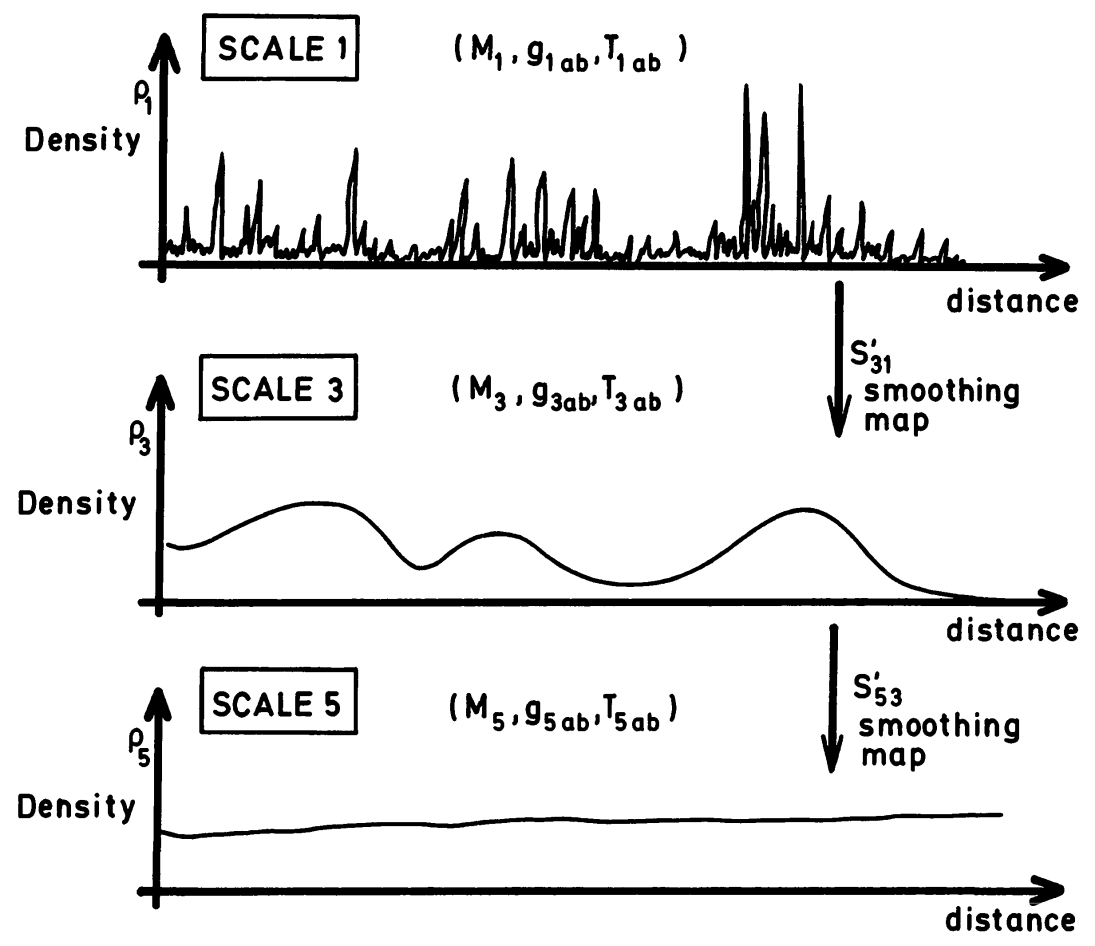

Figure 1. Schematic of a smoothing map, reproduced from [1] with kind permission from Springer Science and Business Media. Original caption: A comparison of models of the same region of the universe on three scales showing different amounts of detail. Scale 1 represents all details down to stars. Scale 3 represents all details down to galaxies. Scale 5 represents only large scale features. The models at different scales are related by (1) identification of which points at the different scales correspond, through maps $S^{*} ;(2)$ maps $S$ determining the matter tensor and maps $S^{\prime}$ determining the metric tensor of the smoothed-out representation from the more detailed one. The usual models of cosmology are at Scale 5; but models for galaxy formation must be at Scale 3 .

extremely close to a metric, $g_{a b}^{(0)}$, with FLRW symmetry-except in the immediate vicinity of very strong field objects, such as neutron stars and black holes $\$$ and is easily represented as such. Thus, the "fitting problem" for $g_{a b}$ is trivially solved. On the other hand, as noted above, the actual stress-energy tensor $T_{a b}$ of our universe is very far from possessing FLRW symmetry. Nevertheless, since $g_{a b}$ is so close to $g_{a b}^{(0)}$, there is no difficulty in defining a suitable large scale average of $T_{a b}$, using either $g_{a b}$ or $g_{a b}^{(0)}$. So, the first question above poses no difficulty.

However, the second question is less trivial. Although order of magnitude estimates clearly indicate that $t_{a b}^{(0)}$ should be negligible for our universe [3], one would like to have a

$\ddagger$ The large deviation of $g_{a b}$ from $g_{a b}^{(0)}$ in the immediate vicinity of strong field objects is irrelevant to cosmology. This is most easily seen by considering a new metric, $g_{a b}^{\prime}$, corresponding to a universe in which each of the strong field objects is replaced by an object of the same mass but having a radius of $\sim 10^{4}$ Schwarzschild radii. Then $g_{a b}^{\prime}$ would be extremely close to $g_{a b}^{(0)}$ everywhere, but the cosmological properties of $g_{a b}$ and $g_{a b}^{\prime}$ would be indistinguishable. 
controlled approximation scheme, wherein the dominant corrections to $g_{a b}^{(0)}$ arising from $t_{a b}^{(0)}$ can be computed. We have recently developed such an approximation scheme [4], wherein the actual metric $g_{a b}$ is assumed to be close to a smooth metric $g_{a b}^{(0)}$, but derivatives of $g_{a b}$ need not be close to derivatives of $g_{a b}^{(0)}$, and second derivatives (i.e., the curvature) of $g_{a b}$ may have unbounded fluctuations relative to $g_{a b}^{(0)}$. A priori, our approach allows for significant backreaction effects, since the dominant contribution to $t_{a b}^{(0)}$ comes from quadratic products of first spacetime derivatives of metric perturbations, which are not assumed to be small. Our main result was the proof that-provided the matter stress-energy tensor satisfies the weak energy condition $-t_{a b}^{(0)}$ must be traceless and must itself satisfy the weak energy condition. Essentially, $t_{a b}^{(0)}$ can be large only in the presence of significant amounts of gravitational radiation, in accord with standard intuition. There cannot be any large backreaction effects arising from matter inhomogeneities. We will describe our approach and summarize the main results of [4] in section 2

Even though no large backreaction effects can occur due to deviations from an FLRW model on small scales, it is still of interest to know exactly what the corrections due to backreaction are. In particular, simulations of structure formation are done using Newtonian cosmological models. Exactly what "dictionary" should be used to translate Newtonian simulations into general relativistic cosmological models? Exactly how accurate is this dictionary, particularly on large scales? We have answered these questions in [5] using a "counting scheme" based upon our approximation method, and we will summarize our main results in section 4. We also include in that section a brief discussion of the degree to which observable quantities in our universe can differ from an FLRW model.

The above results enable us to provide the following answer to the question posed in the title of our paper: The metric of our universe is extremely well approximated by an FLRW metric that satisfies (1) with negligible $t_{a b}^{(0)}$. Certain observable quantities involving geodesics (e.g., gravitational lensing) and curvature (e.g., density contrasts) can differ very significantly from an FLRW model, but these can be accurately computed from Newtonian cosmological models using a dictionary of appropriate accuracy, as described in section 4 .

Before describing our results in the following sections, we now elucidate the nature of the "fitting problem" for our universe by exploring more fully the ball bearing analogy alluded to above. Consider a convex polyhedron with 30,000 faces, where the faces are taken to be tangent to randomly chosen points on a unit sphere. We cannot easily draw such a figure with the required resolution, so to illustrate the kind of surface we have in mind, we have drawn such a polyhedron with 300 faces in figure 2, but it should be kept in mind that the polyhedron we are considering has a hundred times as many faces as the one drawn in figure 2. For a polyhedron with 30,000 faces of the sort that we are considering, the typical linear size of each face is $\sim 1 / 50$ of the radius of the sphere, and the typical deviation of the polyhedral surface from the unit sphere is no more than about 1 part in $10^{4}$. Thus, a solid steel body with this polyhedral surface would pass 

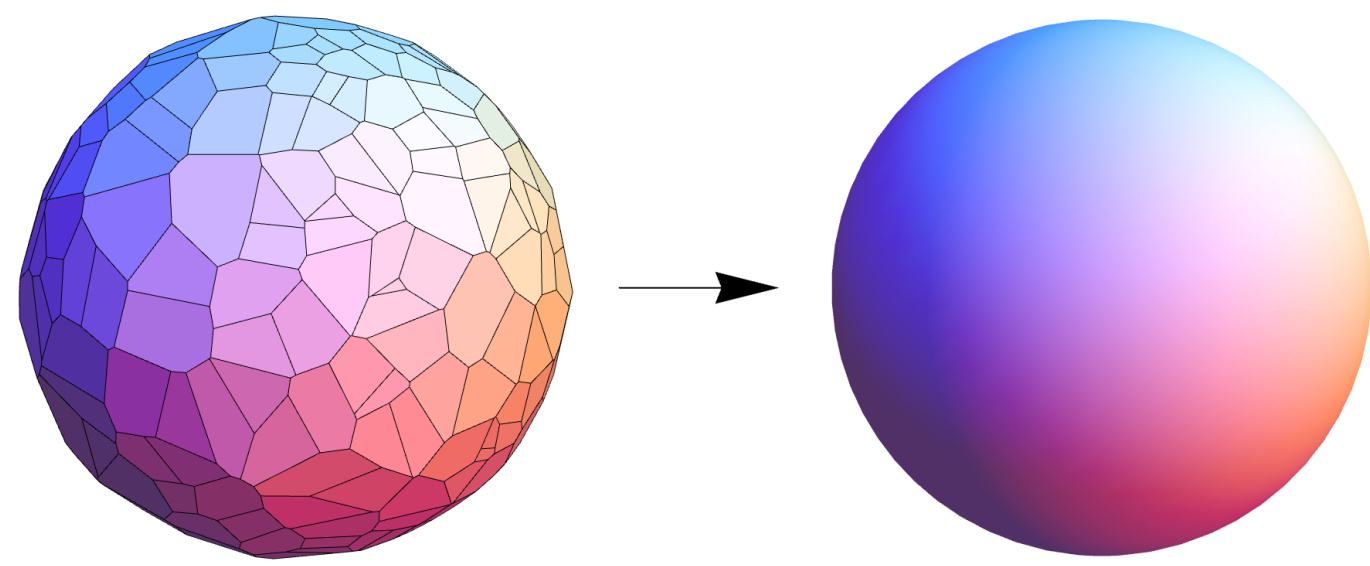

Figure 2. The "fitting problem" is analogous to the problem of approximating the surface of a ball bearing by a sphere. Left: A 300-face convex polyhedron constructed by intersecting the planes tangent to 300 randomly chosen points on the surface of a sphere. Our "Sphereland" is constructed by starting with a similar polyhedron with 100 times as many faces, and slightly rounding the vertices and edges. Right: A sphere that solves the "fitting problem" for the polyhedron (compare with figure 1). Note that we consider a 2-dimensional surface for illustrative purposes only. In higher dimensions, the polygons must be replaced by higher dimensional polyhedra and the number of polyhedra would have to be corrected, but the construction would be otherwise identical.

inspection as a semi-precision ball bearing (see [6]).

The curvature of our polyhedral surface is concentrated as $\delta$-functions at the vertices, and the metric of the polyhedron also fails to be smooth at the edges. We therefore slightly round the vertices and edges, to produce a smooth surface, with large curvature near the (former) vertices and nearly flat geometry elsewhere.

In the spirit of George Gamow's discussion of "Flatland" in his book One, Two, Three ... Infinity [7], we now consider how creatures making observations on our above constructed "Sphereland" would interpret what they experience. Our observers are assumed to be tiny - much smaller than the typical distance between the vertices of the original polyhedron. They are blind, and they cannot move off the surface. Nevertheless, they have ropes that they can pull tightly between any two points on the surface, and they can measure rope lengths and angles between ropes extremely accurately. This allows them to accurately map out the curvature of Sphereland by measuring the deviation from $180^{\circ}$ of the sum of the angles of a triangle constructed from taut ropes. When they plot the curvature, they obtain results that depend upon the size of the triangle they use, in a manner that qualitatively looks very much like figure 1 . In particular, when they use triangles that are much smaller than the typical distance between the vertices, the curvature fluctuates enormously, usually being very small but occasionally being extremely large. On the other hand, when they use triangles that are large compared with the distance between vertices, they obtain results that 
are reasonably consistent with spherical geometry. Nevertheless, even when looking at phenomena on large scales, the observers find some disturbing anomalies when attempting to model Sphereland by a perfect sphere. For example, when they measure the angle subtended by a distant object (by stretching ropes to the ends of the object and measuring the angle between the ropes), they usually find it to be somewhat smaller than predicted by the sphere model, but occasionally they find it to be much larger. They also find that they can sometimes span more than one taut rope between the same two points, even though these points are not close to being antipodal in the sphere model.

As a result of these observations, the observers might be tempted to conclude that the perfect sphere model provides a reasonably good description of Sphereland on large scales - although with some significant deviations - but an extremely poor description of Sphereland on small scales. However, the actual situation, of course, is that the metric, $q_{a b}$, of Sphereland is everywhere extremely close to the metric, $q_{a b}^{(0)}$, of a perfect sphere. This is true on all scales and does not require any averaging of the Sphereland metric. Nevertheless, despite the fact that $q_{a b}$ and $q_{a b}^{(0)}$ are extremely close, their derivatives are not very close, so, in particular, geodesics determined by $q_{a b}$ can differ significantly from geodesics determined by $q_{a b}^{(0)}$. This accounts for the angular size and other anomalies they find in their large scale measurements. Finally, second derivatives of $q_{a b}$ differ enormously from second derivatives of $q_{a b}^{(0)}$, so the curvature of $q_{a b}$ differs enormously from that of $q_{a b}^{(0)}$. In summary, the geometry of Sphereland is described by a metric of the form $q_{a b}^{(0)}+s_{a b}$, where $q_{a b}^{(0)}$ is the metric of a perfect sphere and $\left|s_{a b}\right| \ll\left|q_{a b}^{(0)}\right|$, but first derivatives of $s_{a b}$ are not small, and second derivatives of $s_{a b}$ may be enormous.

In an exactly similar manner, the spacetime metric of our universe takes the form $\S$ $g_{a b}=g_{a b}^{(0)}+\gamma_{a b}$, where $g_{a b}^{(0)}$ has FLRW symmetry and the components of $\gamma_{a b}$ are extremely small relative to $g_{a b}^{(0)}$ - at the level of at most about 1 part in $10^{4}$. This is true on all scales - except in the immediate vicinity of black holes and neutron star: $\llbracket$ and does not require any averaging. However, first derivatives of $\gamma_{a b}$ are not negligibly small, and second derivatives of $\gamma_{a b}$ can be enormous, in accord with the large density inhomogeneities that we observe on scales much less than the Hubble radius.

$\S$ It is fair to ask how we "know" the facts asserted in this paragraph. As with all scientific "knowledge," our beliefs are based on having a small set of simple assumptions that account for a vast amount of disparate data in a mathematically consistent manner. The $\Lambda$ CDM model is based upon a simple set of assumptions and successfully accounts for a vast amount of disparate data. The results summarized in this article confirm that it is mathematically consistent. Our figure of " 1 part in $10^{4}$ " comes from Newtonian cosmological simulations, which yield values of the Newtonian potential in the present universe no larger than $\sim 10^{-4}$ (as occurs near the center of the richest galaxy clusters); our dictionary (see section 4) implies that the spacetime metric deviations from FLRW are of the same size as the Newtonian potential.

|| Korzyński [8] has recently extended the Lindquist-Wheeler universe consisting of a lattice of black holes [9] to irregular black hole distributions, and has shown that an FLRW metric arises as a continuum limit as the number of black holes goes to infinity. This construction illustrates that even in a universe containing only black holes, the metric can be very close to an FLRW metric except in the immediate vicinity of the black holes. 
Nevertheless, the fact that $g_{a b}$ is very close to $g_{a b}^{(0)}$ does not imply that backreaction effects of inhomogeneities must be negligible; as already discussed above, a priori the quantity $t_{a b}^{(0)}$ appearing in 1 could be large. In the next section, we will summarize our results showing that, in fact, matter inhomogeneities cannot produce large backreaction effects. In section 3 we will discuss the main flaw of some other approaches that have suggested that large backreaction effects may occur. In section 4 , we will describe how to very accurately translate Newtonian cosmological models into general relativistic models.

Our notation follows [10]. Roman letters from the early alphabet, $\{a, b, c, \ldots\}$, denote abstract spacetime indices. Roman letters from mid-alphabet, $\{i, j, k, \ldots\}$, denote spatial indices.

\section{Summary of our framework and results}

In this section we briefly review the framework of [4] and summarize our main results. Our framework is a generalization to the non-vacuum case of a framework proposed by Burnett [11], which itself is a rigorous version of Isaacson's approach [12, 13]. Related approaches that make Newtonian assumptions from the outset are given in [14-16]; see also [17].

As indicated in the Introduction, we are interested in describing a situation where the actual spacetime metric is of the form $g_{a b}=g_{a b}^{(0)}+\gamma_{a b}$, where $g_{a b}^{(0)}$ has FLRW symmetry and $\gamma_{a b}$ is small, but where first derivatives of $\gamma_{a b}$ are not small and second derivatives of $\gamma_{a b}$ may be unboundedly large. The FLRW symmetry of $g_{a b}^{(0)}$ plays no role in our framework or results, so in this section, $g_{a b}^{(0)}$ may be any smooth metric.

We would like to determine what restrictions Einstein's equation places on $g_{a b}^{(0)}$. To do so, we could try to proceed by plugging the metric form $g_{a b}=g_{a b}^{(0)}+\gamma_{a b}$ into Einstein's equation and collecting terms of the various "sizes." The largest terms are those that are linear in the second derivatives of $\gamma_{a b}$ and do not have any other factors of $\gamma_{a b}$. However, these terms become negligibly small when averaged. The next largest terms are of two distinct types: (a) terms made from $g_{a b}^{(0)}$ alone and (b) terms quadratic in $\gamma_{a b}$ that have a total of two derivatives acting on $\gamma_{a b}$. When averaged over a spacetime region that is small compared with the radius of curvature of $g_{a b}^{(0)}$ but large compared with the variation scale of $\gamma_{a b}$, we will get an equation of the form $(1)$, where $t_{a b}^{(0)}$ is the average of the quadratic terms (b). Since all terms in Einstein's equation contain at most a total of only two derivatives acting on the metric, all of the cubic and higher order terms in $\gamma_{a b}$ appearing in Einstein's equation become negligible when averaged.

However, if one tries to work with a finite perturbation $\gamma_{a b}$ in the above manner, it would be difficult to precisely define the "averaging" that must be done to obtain to obtain (1), as well as to justify various manipulations of terms. It would also be extremely difficult to obtain any precise, general results on the properties of $t_{a b}^{(0)}$. In order to have precise mathematical rules and obtain precise results, we would like to work with "infinitesimal" $\gamma_{a b}$. As in ordinary perturbation theory (see, e.g., section 7.5 of 
[10]), this means working with suitable one-parameter families of metrics $g_{a b}(\lambda)$ such that $\left[g_{a b}(\lambda)-g_{a b}^{(0)}\right] \rightarrow 0$ as $\lambda \rightarrow 0$. However, in order to represent the sort of perturbations that we are interested in, we do not want to require that spacetime derivatives of $\left[g_{a b}(\lambda)-g_{a b}^{(0)}\right]$ go to zero as $\lambda \rightarrow 0$. Our precise assumptions on $g_{a b}(\lambda)$ are as follows [4]:

(i) Einstein's equation holds for all $\lambda>0$, i.e., we have

$$
G_{a b}(g(\lambda))+\Lambda g_{a b}(\lambda)=8 \pi T_{a b}(\lambda),
$$

where $T_{a b}(\lambda)$ satisfies the weak energy condition, i.e., for all $\lambda>0$ we have $T_{a b}(\lambda) t^{a}(\lambda) t^{b}(\lambda) \geq 0$ for all vectors $t^{a}(\lambda)$ that are timelike with respect to $g_{a b}(\lambda)$.

(ii) There exists a smooth positive function $C_{1}(x)$ on $M$ such that

$$
\left|\gamma_{a b}(\lambda, x)\right| \leq \lambda C_{1}(x)
$$

where $\gamma_{a b}(\lambda, x) \equiv g_{a b}(\lambda, x)-g_{a b}(0, x)$.

(iii) There exists a smooth positive function $C_{2}(x)$ on $M$ such that

$$
\left|\nabla_{c} \gamma_{a b}(\lambda, x)\right| \leq C_{2}(x)
$$

(iv) There exists a smooth tensor field $\mu_{\text {abcdef }}$ on $M$ such that

$$
\underset{\lambda \rightarrow 0}{\mathrm{w}-\lim _{\lambda \rightarrow 0}}\left[\nabla_{a} \gamma_{c d}(\lambda) \nabla_{b} \gamma_{e f}(\lambda)\right]=\mu_{a b c d e f}
$$

where "w-lim" denotes the weak limit.

Here the norms appearing in assumptions (ii) and (iii) are taken with respect to any fixed (i.e., $\lambda$-independent) Riemannian metric on spacetime and $\nabla_{a}$ denotes any fixed derivative operator. The notion of "weak limit", which appears in assumption (iv), corresponds roughly to taking a local spacetime average, and then taking the limit as $\lambda \rightarrow 0$. More precisely, $A_{a_{1} \ldots a_{n}}(\lambda)$ is said to converge weakly to $A_{a_{1} \ldots a_{n}}^{(0)}$ as $\lambda \rightarrow 0$ if and only if, for all smooth tensor fields $f^{a_{1} \ldots a_{n}}$ of compact support,

$$
\lim _{\lambda \rightarrow 0} \int f^{a_{1} \ldots a_{n}} A_{a_{1} \ldots a_{n}}(\lambda)=\int f^{a_{1} \ldots a_{n}} A_{a_{1} \ldots a_{n}}^{(0)} .
$$

Assumptions (ii) and (iii) capture the notion that $\gamma_{a b}$ is small but its first spacetime derivatives need not be small. Assumption (iv) captures the notion that local spacetime averages of quadratic products of first derivatives of $\gamma_{a b}$ are well behaved. An explicit example of a one parameter family satisfying assumptions (ii)-(iv) is given in [18].

Assumptions (i)-(iv) allow us to rigorously derive an equation satisfied by $g_{a b}^{(0)}$ of the form (1), where $t_{a b}^{(0)}$ is given by an explicit formula in terms of $\mu_{a b c d e f}$. In [4], we then proved two theorems constraining $t_{a b}^{(0)}$ :

Theorem 1 Given a one-parameter family $g_{a b}(\lambda)$ satisfying assumptions (i) $-(i v)$ above, the effective stress-energy tensor $t_{a b}^{(0)}$ appearing in equation (1) for the background metric $g_{a b}^{(0)}$ is traceless,

$$
t^{(0) a}=0 .
$$


Theorem 2 Given a one-parameter family $g_{a b}(\lambda)$ satisfying assumptions (i)-(iv) above, the effective stress-energy tensor $t_{a b}^{(0)}$ appearing in equation (1) for the background metric $g_{a b}^{(0)}$ satisfies the weak energy condition, i.e.,

$$
t_{a b}^{(0)} t^{a} t^{b} \geq 0
$$

for all $t^{a}$ that are timelike with respect to $g_{a b}^{(0)}$.

In essence, these theorems show that only those small scale metric inhomogeneities corresponding to gravitational radiation can have a significant backreaction effect; see [4] for further discussion. In particular, it should be noted that in the case where $g_{a b}^{(0)}$ has FLRW symmetry, even when short wavelength gravitational radiation is present and backreaction effects are large, the effective stress-energy tensor must be of the form of a $P=\frac{1}{3} \rho$ fluid, and therefore cannot mimic dark energy.

In section 4, we will return to the issue of how best to describe $g_{a b}$ in cosmology. Specifically, we will consider how to translate Newtonian cosmologies into general relativistic cosmologies in a way that yields accurate solutions to Einstein's equation. However, we first give a brief discussion of some other approaches that have suggested that backreaction might be large.

\section{Other approaches to backreaction}

As the results summarized in the previous section have shown, small scale density inhomogeneities cannot produce large backreaction effects in cosmology. However, a number of other approaches have come to the opposite conclusion - or, at least, have suggested the possibility that the cosmological backreaction effects due to matter inhomogeneities on small and/or large scales could be large. Some of these approaches are based upon questionable approximation methods (see, e.g., [19]) and some are based upon questionable interpretations of gauge-dependent quantities (see, e.g., [20 24] with regard to long wavelength perturbations); we will not attempt to review such approaches here. However, other approaches proceed by assigning an FLRW metric, $g_{a b}^{(0)}$, to $g_{a b}$ via some averaging procedure or the matching of some observables. As we shall now illustrate, the main flaw with such approaches is that the FLRW metric $g_{a b}^{(0)}$ that one thereby obtains may not be close to $g_{a b}$ - even in cases where $g_{a b}$ is well approximated by some FLRW metric. Since $g_{a b}^{(0)}$ may be a poor approximation to $g_{a b}$, there is no reason why $g_{a b}^{(0)}$ need be close to a solution to Einstein's equation, so one may obtain large backreaction terms in equations satisfied by $g_{a b}^{(0)}$. Of course, these apparent backreaction effects are entirely spurious, being generated by using a poor approximation to $g_{a b}$.

The approach of Buchert [25, 26] provides a good illustration of this point. In Buchert's approach the metric is written in a synchronous slicing comoving with the dust particles,

$$
d s^{2}=-d t^{2}+{ }^{(3)} g_{i j}(t) d x^{i} d x^{j} .
$$


This is possible provided the dust 4 -velocity $u^{a}$ is irrotational, which we assume here. The volume, $V_{\mathcal{D}}$, of a comoving spatial region $\mathcal{D}$ on a constant- $t$ slice $\Sigma_{t}$ is given by

$$
V_{\mathcal{D}}(t)=\int_{\mathcal{D}} \mathrm{d} \Sigma_{t}
$$

where $\mathrm{d} \Sigma_{t}=\sqrt{{ }^{(3)} g(t)} \mathrm{d}^{3} x$ is the proper volume element on $\Sigma_{t}$. The averaged "scale factor," $a_{\mathcal{D}}(t)$, is defined as proportional to $V_{\mathcal{D}}^{1 / 3}$. Spatial averages of scalars $\psi$ over $\mathcal{D}$ can then be defined by

$$
\langle\psi\rangle_{\mathcal{D}}(t) \equiv \frac{1}{V_{\mathcal{D}}} \int_{\mathcal{D}} \psi \mathrm{d} \Sigma_{t}
$$

In this way, one can define an averaged density, $\langle\rho\rangle_{\mathcal{D}}(t)$, and averaged scalar curvature, $\left\langle{ }^{(3)} \mathcal{R}\right\rangle_{\mathcal{D}}(t)$. One may then assign an FLRW model, $g_{a b}^{(0)}$, to $g_{a b}$ based upon the values of these averaged quantities.

Equations governing the dynamics of $g_{a b}^{(0)}$ can be derived by taking averages of the scalar components of Einstein's equation for $g_{a b}$. One obtains [25, 26]

$$
\begin{aligned}
& 3\left(\frac{\dot{a}_{\mathcal{D}}}{a_{\mathcal{D}}}\right)^{2}=8 \pi\langle\rho\rangle_{\mathcal{D}}-\frac{1}{2}\left\langle{ }^{(3)} \mathcal{R}\right\rangle_{\mathcal{D}}-\frac{1}{2} \mathcal{Q}_{\mathcal{D}}, \\
& 3 \frac{\ddot{a}_{\mathcal{D}}}{a_{\mathcal{D}}}=-4 \pi\langle\rho\rangle_{\mathcal{D}}+\mathcal{Q}_{\mathcal{D}}
\end{aligned}
$$

where the quantity

$$
\mathcal{Q}_{\mathcal{D}} \equiv \frac{2}{3}\left(\left\langle\theta^{2}\right\rangle_{\mathcal{D}}-\langle\theta\rangle_{\mathcal{D}}^{2}\right)-2\left\langle\sigma^{2}\right\rangle_{\mathcal{D}}
$$

characterizes the "backreaction," i.e., the failure of the FLRW spacetime $g_{a b}^{(0)}$, to satisfy

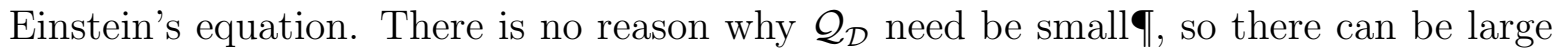
backreaction within the Buchert framework.

The large backreaction that can occur in the Buchert framework is a direct consequence of the fact that $g_{a b}^{(0)}$ can be a poor approximation to $g_{a b}$. This is most easily seen in the case where $g_{a b}$ is not globally close to a single FLRW metrid + A nice example of this is provided by a universe that consists of two disconnected dust FLRW universes in different stages of expansion. The Buchert prescription would represent this disconnected universe as a single FLRW universe, which provides a bad approximation to the actual metric everywhere. One can give an example of this sort [3] wherein the "backreaction" is so large that one obtains acceleration of the representative FLRW universe, even though each of the disconnected components of the actual universe is

I A further difficulty with the Buchert approach is that the equations are underdetermined, as there is no evolution equation given for $\mathcal{Q}_{\mathcal{D}}$. However, one is not free to specify $\mathcal{Q}_{\mathcal{D}}(t)$ arbitrarily, as the exact (i.e., not averaged) metric must satisfy the Einstein equation with dust stress energy, but only two components of this equation have been used to get the Buchert equations. See [18 for further discussion.

+ If the actual metric, $g_{a b}$, of the universe were not globally close to a single FLRW metric, we do not believe that it would be useful to introduce any concept whatsoever of an "averaged" FLRW metric $g_{a b}^{(0)}$. 
decelerating. Obviously, this acceleration is not a physical effect but is a spurious artifact of the poor approximation that $g_{a b}^{(0)}$ provides to the actual spacetime metric.

However, the Buchert prescription can also give a poor approximation to the actual spacetime metric $g_{a b}$-and, correspondingly, yield a large backreaction - even when $g_{a b}$ is well approximated by (or even equal to!) some FLRW metric. An example of this is provided by Minkowski spacetime, which has exact FLRW symmetry. Let $(T, X, Y, Z)$ be global inertial coordinates and consider the hypersurface defined by

$$
T=f(X, Y, Z),
$$

with

$$
f(X, Y, Z)=\frac{1}{3 N} \sin (N X) \sin (N Y) \sin (N Z),
$$

with $N$ large. This hypersurface is extremely close to the hypersurface $T=0$, but is extremely "wiggly." (However, note that $\left|\nabla_{i} f\right|<1$ everywhere, so the hypersurface is everywhere spacelike.) We choose this hypersurface as the initial hypersurface of a synchronous coordinate system (9). We take the domain, $\mathcal{D}$, in the initial hypersurface to be a coordinate cube of side $[0,2 \pi]$. Since the matter stress-energy vanishes, we obviously have $\langle\rho\rangle_{\mathcal{D}}=0$. However, we have verified numerically that $\left\langle{ }^{(3)} \mathcal{R}\right\rangle_{\mathcal{D}}$ is enormous for sufficiently large $N$. Thus, although the actual metric, $g_{a b}$, of Minkowski spacetime has exact FLRW symmetry, the Buchert procedure with the above choice of hypersurface instructs us to represent the Minkowski metric with an "averaged" FLRW metric $g_{a b}^{(0)}$ that is an extremely poor approximation to $g_{a b}$ on all scales. The backreaction term $\mathcal{Q}_{\mathcal{D}}$ is correspondingly large. Again, this backreaction is entirely spurious.

One might object to the above example on the grounds that there is no matter present in Minkowski spacetime and we have arbitrarily chosen some artificial hypersurface on which to perform the Buchert construction. However, we could modify this example by adding a tiny sprinkling of dust following geodesics orthogonal to our hypersurface. If the mass density of the dust is sufficiently small, the metric will be essentially unaffected by the dust, but the Buchert procedure will require us to use the above hypersurface and will provide us with an effective FLRW metric that is a very poor approximation to the actual spacetime metric. Of course, the velocity distribution of the dust in this example is not representative of any physically realistic situation. But the example is useful for showing that the Buchert construction depends upon the velocity distribution of the dust even when the dust makes a negligible contribution to the stress-energy. In realistic cosmological situations, the perturbations to the dust velocity will be much larger than the perturbations to the metric (see subsection 4.2 below), and the comoving synchronous hypersurfaces of the Buchert construction will provide a poor choice for approximating the hypersurfaces with nearly FLRW symmetry. This will lead to a correspondingly large backreaction. We will comment further on this point at the end of subsection 4.2 .

For similar reasons, large backreaction also appears in the approach of Clarkson and Umeh [27]. These authors obtain expressions for an effective $H$ and $q_{0}$-and, thereby, a representative FLRW metric $g_{a b}^{(0)}$ - by writing down an approximate formula for the 
angular diameter distance $d_{\mathrm{A}}$ as a function of redshift $z$ and performing an average. However, lensing effects are sensitive to small scale structure since they depend on derivatives of the metric; as discussed further in subsection 4.3 below, lensing effects can be very large even when the deviations of the metric from an FLRW metric are very small. Consequently, for the power spectra they consider, they obtain UV divergent expressions for their effective $H$ and $q_{0}$. The expressions for backreaction are similarly divergent. Again, these large backreaction effects arise from the fact that their choice of FLRW metric, $g_{a b}^{(0)}$, is an extremely poor approximation ${ }^{*}$ to the actual spacetime metric, $g_{a b}$.

\section{Cosmology with a Newtonianly-perturbed FLRW metric}

As we have argued above, the metric of the universe is well described by a metric of the form $g_{a b}=g_{a b}^{(0)}+\gamma_{a b}$, where $g_{a b}^{(0)}$ is an FLRW metric satisfying the usual Einstein equation with the averaged matter stress-energy as source, and $\gamma_{a b}$ is "small" - although its derivatives are not small. We would like to determine $\gamma_{a b}$ more precisely. It is clear that $\gamma_{a b}$ does not merely satisfy the linearized Einstein equation about $g_{a b}^{(0)}$, since nonlinear terms in derivatives of $\gamma_{a b}$ cannot be ignored. In particular, we would like to know the leading order effects that these nonlinear corrections have on large scale behavior, including the expansion rate of the universe.

We analyzed this issue in [4] in the context of the completely general framework described in section 2 above. In that reference, we derived an equation satisfied by the "long wavelength" part of $\gamma_{a b}$ that takes into account the leading order nonlinear corrections due to the small scale inhomogeneities. However, this equation is extremely complicated, and it is not easy to see what effects it predicts in a general context. To make further progress, it is necessary to put in more assumptions about the nature of our universe. A key observational fact about our universe is that it is locally Newtonian, i.e., as seen by observers "at rest" with respect to $g_{a b}^{(0)}$, velocities of nearby objects are nonrelativistic and their dynamics is well described by Newtonian gravity - except, of course, in the immediate vicinity of black holes and neutron stars, which are not relevant for this discussion. Indeed, cosmologists normally simulate structure formation in our universe using only Newtonian gravity - even on scales comparable to the Hubble radius!

These considerations allow us to reformulate the issue of determining the leading order corrections to $g_{a b}^{(0)}$ as follows [5]: Consider a cosmological solution to the equations of Newtonian gravity of the kind produced in numerical simulations by cosmologists. We may ask the following questions: (1) What "dictionary" should be used to transcribe this Newtonian cosmology into a general relativistic cosmology? (2) How accurate is

* It may sound odd that an FLRW metric $g_{a b}^{(0)}$ that is chosen to be the best fit to some observation made in the nearly FLRW metric, $g_{a b}$, should be an extremely poor approximation to $g_{a b}$. However, one might note that if our Sphereland observers chose the sphere metric that gave the best fit to their observation of, e.g., the average of the square of the curvature, they would get an extremely poor approximation to the metric of Sphereland. 
this dictionary, i.e., how close does the resulting general relativistic cosmology come to satisfying Einstein's equation? Of course, these questions are not independent: If the dictionary did not produce accurate results, this would indicate that we need a better dictionary. As we shall describe below, there is a straightforward dictionary suggested by the correspondence between linearized Newtonian gravity and linearized general relativity. This dictionary is quite satisfactory, but it can be further improved [5] to take into account post-Newtonian effects at small scales as well as the leading order large scale effects produced by the nonlinear effects of inhomogeneities. The resulting improved dictionary should yield an extremely accurate general relativistic description of our universe, and, in particular, it provides the leading order backreaction effects at large scales produced by small scale inhomogeneities.

We will now review Newtonian cosmology. We will then present a dictionary that maps Newtonian solutions into general relativistic cosmologies and discuss its accuracy. Finally we discuss some effects that these inhomogeneities have on light propagation and observables.

\subsection{Newtonian cosmology}

It is a remarkable fact that the evolution equations for a homogeneous and isotropic cosmology with dust matter and a cosmological constant are precisely the same in Newtonian gravity and general relativity. To see this, begin with the equations of Newtonian gravity with a cosmological constant,

$$
\begin{aligned}
& \partial^{i} \partial_{i} \phi+\Lambda=4 \pi \rho, \\
& \partial_{t} \rho+\partial_{i}\left(\rho v^{i}\right)=0, \\
& \partial_{t}\left(\rho v^{i}\right)+\partial_{j}\left(\rho v^{i} v^{j}\right)=-\rho \partial^{i} \phi .
\end{aligned}
$$

Plugging in the cosmological ansatz, $\rho=\rho_{0}(t), v^{i}=H(t) x^{i}$, and requiring solutions to be non-singular, it can be seen [28] that (17)-(19) reduce to the Friedmann equations for a dust cosmology,

$$
\begin{aligned}
& \frac{d H}{d t}+H^{2}=-\frac{4 \pi}{3} \rho_{0}+\frac{\Lambda}{3}, \\
& H^{2}=\frac{8 \pi}{3} \rho_{0}+\frac{\Lambda}{3}-\frac{k}{a^{2}} .
\end{aligned}
$$

Here $a$ is the radius of any comoving ball, so that

$$
H=\frac{1}{a} \frac{d a}{d t} .
$$

The constant $k$ is a constant of integration, and the density $\rho_{0}$ varies as $1 / a^{3}$. Thus, there is an obvious correspondence between Newtonian and relativistic dust cosmologies in the homogeneous and isotropic case, which takes exact solutions to exact solutions. In the following, we restrict consideration to the $k=0$ case.

It is useful to rewrite the exact Newtonian equations relative to a background solution and to transform to a comoving coordinate system of the background. We 
obtain [5]

$$
\begin{aligned}
& \partial^{i} \partial_{i} \psi_{\mathrm{N}}=4 \pi a^{2} \rho_{0} \delta_{\mathrm{N}}, \\
& \dot{\delta}_{\mathrm{N}}+\partial_{i}\left(\left(1+\delta_{\mathrm{N}}\right) v_{\mathrm{N}}^{i}\right)=0, \\
& \dot{v}_{\mathrm{N}}^{i}+v_{\mathrm{N}}^{j} \partial_{j} v_{\mathrm{N}}^{i}+\frac{\dot{a}}{a} v_{\mathrm{N}}^{i}=-\partial^{i} \psi_{\mathrm{N}},
\end{aligned}
$$

where $v_{\mathrm{N}}^{i}$ is the velocity relative to the "Hubble flow," $\psi_{\mathrm{N}}$ is the Newtonian potential relative to the background, and $\delta_{\mathrm{N}}$ is the fractional density perturbation. We have inserted the subscript " $\mathrm{N}$ " on these expressions in order to be able to clearly distinguish them from the corresponding general relativistic quantities that we will consider below. Equations (23)-(25) (or variants describing discrete matter sources) form the basis of numerical simulations exploring structure formation in cosmology.

\subsection{Mapping to general relativity}

It is perhaps an even more remarkable fact that the exact correspondence between homogeneous, isotropic Newtonian and general relativistic dust cosmologies also extends to linearized solutions in the $k=0$ case, i.e., linearized solutions of (23)-25) correspond exactly to (scalar and vector sector) solutions of the $k=0$ general relativistic perturbation equations [29] when an appropriate "dictionary" is used. For scalar perturbations, this is most easily seen by noting that, when written in terms of Bardeen's gauge invariant variables, the Einstein equation with dust matter, linearized off of FLRW, is identical to the linearized form of (23)-(25), with the identifications

$$
\begin{aligned}
& \psi_{\mathrm{N}} \longleftrightarrow \Phi_{\mathrm{A}}=-\Phi_{\mathrm{H}}, \\
& v_{\mathrm{N}}^{i} \longleftrightarrow v_{\mathrm{s}}^{i} \\
& \delta_{\mathrm{N}} \longleftrightarrow \epsilon_{\mathrm{m}},
\end{aligned}
$$

where we have used Bardeen's notation for the gauge invariant variables $\Phi_{\mathrm{A}}, \Phi_{\mathrm{H}}, v_{\mathrm{s}}^{i}$, and $\epsilon_{\mathrm{m}}$. A similar correspondence holds in the vector sector, although there it is necessary to also solve a Poisson-type equation to obtain the vector-type metric perturbation [5]. The spacetime metric and stress-energy tensor may be straightforwardly reconstructed in any gauge from the gauge invariant variables.

While the gauge invariant Bardeen variables are extremely convenient to use in linear perturbation theory, there are no analogs of them beyond linear order. Thus, in order to attempt to extend the above exact Newtonian-relativistic correspondence for linearized solutions to an approximate Newtonian-relativistic correspondence for exact solutions, we must make a choice of gauge. We choose to work in "longitudinal gauge," where the metric takes the form

$$
d s^{2}=a^{2}(\tau)\left\{-(1+2 A) d \tau^{2}-2 B_{i} d x^{i} d \tau+\left[\left(1+2 H_{\mathrm{L}}\right) \delta_{i j}+h_{i j}\right] d x^{i} d x^{j}\right\},
$$

where $\partial^{i} B_{i}=\partial^{j} h_{i j}=h^{i}{ }_{i}=0$. As argued in [5], it should be possible to make this gauge choice for metrics that are sufficiently close to FLRW metrics. We will comment further on this gauge choice below. 
We could obtain a dictionary by simply transcribing the linearized dictionary to the nonlinear regime, replacing the Bardeen variables in the linearized correspondence with their metric component counterparts in longitudinal gauge. However, we can easily improve this dictionary by slightly modifying the definitions of $v^{i}$ and $B^{i}$ so as to obtain consistency with the nonlinear momentum constraint at small scales. We obtain [5]

$$
\begin{aligned}
& A=-H_{L}=\psi_{\mathrm{N}}, \\
& \left(1+\delta_{\mathrm{N}}\right) v^{i}=\left(1+\delta_{\mathrm{N}}\right)\left(v_{\mathrm{N}}^{i}+B^{i}\right)-\overline{\left(1+\delta_{\mathrm{N}}\right) v_{\mathrm{N}}^{i}}, \\
& \delta=\delta_{\mathrm{N}}-\frac{3}{4 \pi \rho_{0} a^{2}}\left[\left(\frac{\dot{a}}{a}\right)^{2} \psi_{\mathrm{N}}+\frac{\dot{a}}{a} \dot{\psi}_{\mathrm{N}}\right], \\
& \partial^{j} \partial_{j} B^{i}=-\left.16 \pi \rho_{0} a^{2}\left(\left(1+\delta_{\mathrm{N}}\right) v_{\mathrm{N}}^{i}-\overline{\left(1+\delta_{\mathrm{N}}\right) v_{\mathrm{N}}^{i}}\right)\right|_{\mathrm{v}}, \\
& h_{i j}=0 .
\end{aligned}
$$

In these expressions, the overline denotes an average over all of space, while the subscript "v" denotes the "vector part". Note that it is necessary to solve the Poisson equation (33) to obtain $B^{i}$, but this is a straightforward procedure that could be incorporated into numerical simulations. The quantity $B^{i}$ and the corrections to $v^{i}$ will normally be small. If we neglect these terms, we obtain a continuum version of the dictionary developed in [30]. We note, however, that very recent efforts to incorporate $B^{i}$ into Newtonian simulations have suggested that it may be possible to measure the frame dragging effects of $B^{i}$ [31, 32].

If we start with an exact Newtonian cosmology and translate it into a general relativistic cosmology using the above dictionary, we wish to know how well Einstein's equation is satisfied as well as the corrections to the dictionary that would be needed to make Einstein's equation hold to greater accuracy. In order to proceed, we need to have an a priori notion of the "size" of the Newtonian quantities. To assign such orders, we break up each quantity into its ("long wavelength") averaged part and its ("short wavelength") remainder. We introduce a "small parameter" $\epsilon$, and take the long wavelength parts of $\psi_{\mathrm{N}}, v_{\mathrm{N}}$, and $\delta_{\mathrm{N}}$ to be $\mathrm{O}(\epsilon)$. We also take all space and time derivatives of the long wavelength parts of these quantities to be $\mathrm{O}(\epsilon)$. On small scales, where nonlinear motion is important, our assignment of "sizes" is based on usual postNewtonian counting. We take $\psi_{\mathrm{N}}=\mathrm{O}(\epsilon)$ and $v_{\mathrm{N}}=\mathrm{O}\left(\epsilon^{1 / 2}\right)$. Each spatial derivative is assumed to contribute an inverse power of $\epsilon, \partial_{i} \sim \mathrm{O}\left(\epsilon^{-1}\right)$, and each time derivative is assumed to contribute an inverse half-power of $\epsilon, \partial_{0} \sim \mathrm{O}\left(\epsilon^{-1 / 2}\right)$. Consistent with the Newtonian equation (23), we then have $\delta_{\mathrm{N}}=\mathrm{O}\left(\epsilon^{-1}\right)$. Oliynyk has proven existence of a wide class of one-parameter families of inhomogeneous solutions to the Einstein equation with this behavior [33, 34].

With this counting scheme in hand, we can check how well the Einstein equation is satisfied by the relativistic cosmology given by our dictionary. A priori, the long wavelength part of Einstein's equation contains terms at most $\mathrm{O}(\epsilon)$, whereas the short wavelength part of Einstein's equation contains terms that are $\mathrm{O}\left(\epsilon^{-1}\right)$. We find that, with the above dictionary, the long wavelength part fails to hold at $\mathrm{O}(\epsilon)$ - due to the 
appearance of such terms as the average of quadratic products of derivatives of the short wavelength part of $\psi_{\mathrm{N}}$ - whereas the short wavelength part of Einstein's equation fails at $\mathrm{O}(1)$. However, we showed in [5] how to improve our dictionary so that the long wavelength part of Einstein's equation holds at $\mathrm{O}(\epsilon)$, and the short wavelength part of Einstein's equation holds at $\mathrm{O}(1)$. The short wavelength corrections are postNewtonian corrections and are negligible for any present-day observations. The long wavelength corrections essentially correspond to including as source terms in Einstein's equation the effects of the energy and stresses of the Newtonian potential and the kinetic motions. These provide the leading order backreaction effects on large scales of the small scale inhomogeneities. Although these corrections are formally $\mathrm{O}(\epsilon)$ in our counting scheme, they are, in fact, negligible $\sharp$. Thus, while it is very comforting to know that the improved "Oxford dictionary" of [5] exists, the dictionary (30)-(34) should provide an excellent description of our universe. The smallness of Newtonian perturbations guarantees that the universe is well-described by an FLRW model, even though the stress-energy perturbations are very large on small scales.

Finally, we make a comment on our choice of metric form (29). It is extremely important that we did not choose a metric form - such as the comoving synchronous form (9) - that is tied to the matter motion, i.e., geodesics of the actual spacetime. This is because the geodesics of the actual spacetime differ significantly from the geodesics of the background spacetime, so the metric components in, e.g., synchronous coordinates can be very different even when the metrics are actually very close. In the present instance, this phenomenon is manifested as follows: Even at the linear level, when the dictionary (26)-(28) is expressed in a particular gauge, there will be some "mixing" between the velocity, the potential, and the density. For example, in the longitudinal gauge, the relativistic density perturbation $(32)$ involves $\psi_{\mathrm{N}}$. However, in the longitudinal gauge, the dictionary provides metric perturbation variables that consistently remain $\mathrm{O}(\epsilon)$ in our counting scheme. By contrast, in the comoving synchronous gauge, metric components pick up contributions from $v_{\mathrm{N}}$. These cause the metric perturbation to become $\mathrm{O}(1)$ on a timescale of order $\epsilon^{1 / 2}$, thereby potentially leading to large, spurious backreaction effects. This is closely related to the example given near the end of section 3 .

\subsection{Effects on observables}

As we have emphasized throughout this article, the spacetime metric, $g_{a b}$, of our universe is very close to an FLRW metric, $g_{a b}^{(0)}$. However, most things that we observe about the universe involve electromagnetic radiation, which propagates on null geodesics. However, the geodesics of the actual metric $g_{a b}$ are not necessarily very close to corresponding geodesics of $g_{a b}^{(0)}$. Moreover, the time evolution of the shear

\# In fact, the next order correction to the pressure vanishes completely when averaging virialized systems [16; this was recently demonstrated numerically [35. The correction to the energy density corresponds to the inclusion of kinetic energy and Newtonian binding energy. 
and convergence of a bundle of geodesics depends on the Riemann curvature [10] (i.e., second derivatives of the metric), which can be very large. As a result, observables in the actual universe described by $g_{a b}$ can differ considerably from observables computed in the FLRW background spacetime $g_{a b}^{(0)}$.

The redshift-luminosity relation provides a good example of the kinds of differences that can occur for observables, even when $g_{a b}$ and $g_{a b}^{(0)}$ are very close. In an FLRW spacetime, the Weyl curvature vanishes and the Ricci curvature is uniform. However, in the actual universe, most of the matter is clumped into structures such as galaxies. If a beam of light passes through a clump of matter, it will encounter high Ricci curvature and experience large convergence. If it passes near - but not through - a clump of matter, it will encounter only Weyl curvature, producing shear and, eventually, convergence. If it does not pass near any clumps of matter, it will not experience any significant curvature at all. Thus, if the actual universe is sufficiently lumpy, then the light beams from most sources will encounter little curvature and will be dimmed compared with the FLRW spacetime [36]. However, occasionally, the beams will pass through strong curvature, and the sources will be greatly magnified and/or multiply imaged. Thus, large and highly non-Gaussian deviations in the apparent luminosity of standard candle high redshift sources will occur [37, 38].

By flux conservation, the average of the apparent luminosity of sources (including multiple images) must match the FLRW value to a high degree of accuracy. Thus, if one were to plot the mean apparent luminosity of standard candle sources versus redshift, one should obtain good agreement with the underlying FLRW model. However, if one were to plot the mean of a nonlinear function of luminosity - such as the luminosity distanceversus redshift, one could get significant departures from the underlying FLRW values at high redshift, on account of the large, non-Gaussian fluctuations. This illustrates the point that, as a general matter of principle, one should not start at the level of observables and then try to design some effective FLRW metric that captures the behavior of these observables. Rather, one must proceed as follows:

(i) Start with a background FLRW metric that is believed to be close to the actual metric of the universe (except, of course, in the immediate vicinity of strong-field objects such as black holes).

(ii) Determine the corrections to the FLRW metric that describe inhomogeneities. To an excellent approximation, these are the (small) Newtonian corrections described in the previous subsection.

(iii) Calculate observables in the full metric (background plus perturbations).

The $\Lambda$ CDM model has been remarkably successful in carrying out this strategy and thereby making predictions in excellent agreement with all cosmological observations. As we have reviewed in this article, this model is mathematically consistent and fully in accord with general relativity. Unless some observational discrepancy arises in the future, the $\Lambda \mathrm{CDM}$ model deserves to be ranked among the great achievements of modern science. 


\section{Acknowledgments}

This research was supported in part by NSF grant PHY 12-02718 to the University of Chicago, and by NSERC. SRG is supported by a CITA National Fellowship at the University of Guelph, and he thanks the Perimeter Institute for hospitality. Research at Perimeter Institute is supported through Industry Canada and by the Province of Ontario through the Ministry of Research \& Innovation.

\section{References}

[1] Ellis G F R 1984 Relativistic cosmology: its nature, aims and problems General Relativity and Gravitation ed Bertotti B, de Felice F and Pascolini A (D. Reidel Publishing Company) pp 215-288

[2] Ellis G and Stoeger W 1987 Class. Quant. Grav. 4 1697-1729

[3] Ishibashi A and Wald R M 2006 Class. Quant. Grav. 23 235-250 (Preprint gr-qc/0509108)

[4] Green S R and Wald R M 2011 Phys. Rev. D83 084020 (Preprint 1011.4920)

[5] Green S R and Wald R M 2012 Phys. Rev. D85 063512 (Preprint 1111.2997)

[6] Abbott Ball Company Semi-Precision Balls Accessed: 2014-07-27 URL http: //www.abbottball.com/products/semi-precision-balls.php

[7] Gamow G 1947 One, Two, Three ... Infinity (N.Y.: The Viking Press, Inc.)

[8] Korzyński M 2014 Class. Quant. Grav. 31085002 (Preprint 1312.0494)

[9] Lindquist R W and Wheeler J A 1957 Rev. Mod. Phys. 29(3) 432-443 URL http://link.aps.org/doi/10.1103/RevModPhys.29.432

[10] Wald R M 1984 General Relativity (Chicago, IL: University of Chicago Press)

[11] Burnett G A 1989 J. Math. Phys. 30 90-96

[12] Isaacson R A 1968 Phys. Rev. 166 1263-1271

[13] Isaacson R A 1968 Phys. Rev. 166 1272-1279

[14] Futamase T 1996 Phys. Rev. D53 681-689

[15] Carbone C and Matarrese S 2005 Phys. Rev. D71 043508 (Preprint astro-ph/ 0407611)

[16] Baumann D, Nicolis A, Senatore L and Zaldarriaga M 2012 JCAP 1207051 (Preprint 1004.2488)

[17] Zalaletdinov R M 1997 Bull. Astron. Soc. India 25 401-416 (Preprint gr-qc/ 9703016) URL http://hdl.handle.net/2248/2265

[18] Green S R and Wald R M 2013 Phys. Rev. D87 124037 (Preprint 1304.2318)

[19] Kolb E W, Matarrese S and Riotto A 2006 New J. Phys. 8322 (Preprint astro-ph/0506534) 
[20] Mukhanov V F, Abramo L R W and Brandenberger R H 1997 Phys. Rev. Lett. 78 1624-1627 (Preprint gr-qc/9609026)

[21] Abramo L R W, Brandenberger R H and Mukhanov V F 1997 Phys. Rev. D56 3248-3257 (Preprint gr-qc/9704037)

[22] Unruh W 1998 (Preprint astro-ph/9802323)

[23] Geshnizjani G and Brandenberger R 2002 Phys. Rev. D66 123507 (Preprint gr-qc/0204074)

[24] Geshnizjani G and Brandenberger R 2005 JCAP 0504006 (Preprint hep-th/ 0310265)

[25] Buchert T 2000 Gen. Rel. Grav. 32 105-125 (Preprint gr-qc/9906015)

[26] Buchert T 2001 Gen. Rel. Grav. 33 1381-1405 (Preprint gr-qc/0102049)

[27] Clarkson C and Umeh O 2011 Class. Quantum Grav. 28164010 (Preprint 1105.1886)

[28] Peebles P 1980 The large-scale structure of the universe (Princeton, NJ: Princeton University Press)

[29] Bardeen J M 1980 Phys. Rev. D22 1882-1905

[30] Chisari N E and Zaldarriaga M 2011 Phys. Rev. D83 123505 (Preprint 1101.3555)

[31] Bruni M, Thomas D B and Wands D 2014 Phys. Rev. D89 044010 (Preprint 1306.1562)

[32] Adamek J, Daverio D, Durrer R and Kunz M 2013 Phys. Rev. D88 103527 (Preprint 1308.6524)

[33] Oliynyk T A 2014 Phys. Rev. D89 124002 (Preprint 1307.6281)

[34] Oliynyk T A 2014 (Preprint 1406.6104)

[35] Adamek J, Clarkson C, Durrer R and Kunz M 2014 (Preprint 1408.2741)

[36] Dyer C C and Roeder R C 1972 The Astrophysical Journal 174 L115

[37] Holz D E and Wald R M 1998 Phys. Rev. D58 063501 (Preprint astro-ph/ 9708036)

[38] Holz D E 1998 The Astrophysical Journal Letters 506 L1 